\title{
AVES ACUÁTICAS DE LAGUNA CÁHUIL, REGIÓN DE O’HIGGINS, CHILE CENTRAL
}

\author{
Jorge Mella-Romeri $^{*}$ \\ ${ }^{1}$ Laboratorio de Conservación Biológica, Departamento de Ciencias Ecológicas, Facultad de Ciencias, \\ Universidad de Chile, Las Palmeras 3425, Casilla 653, Santiago, Chile. \\ *E-mail: Jorge Mella-Romero, jorgemella@ug.uchile.cl
}

\section{RESUMEN}

Los humedales costeros del centro de Chile son sitios ecológicamente relevantes para las aves, pero en las lagunas de la región de O'Higgins, se sabe poco sobre los ensambles de aves acuáticas. Durante 2015 evalué la riqueza y cantidad de aves acuáticas en la laguna Cáhuil, centro de Chile. Realicé conteos mensuales (un día al mes) de cuatro transectos de línea fija $(200 \mathrm{~m})$ establecidos a lo largo de la playa. Se contaron todas las aves presentes en la orilla de la playa, la superficie del agua y los bancos de arena. Detecté 28 especies de aves, siendo Cygnus melancoryphus, Fulica armillata y Larus dominicanus las más numerosas. Se discute la importancia de realizar muestreos sistemáticos para tener una comprensión más completa de los conjuntos de aves acuáticas en toda la región y para desarrollar acciones de conservación.

Palabras Claves: Avifauna, Chile, Cygnus melancoryphus, Especies Amenazadas

\section{ABSTRACT}

Waterfowl of Cáhuil lagoon, O'Higgins's region, central Chile. Coastal wetlands of central Chile are ecologically relevant sites for birds, but little is known about the waterfowl assemblages in lagoons of O'Higgins's region. During 2015 I evaluated the species richness and abundances of aquatic birds in the Cáhuil lagoon, central Chile. I conducted monthly counts (one-day-month) from four fixed linetransects $(200 \mathrm{~m})$ established along the beach. All birds present on beach's shore, water surface and sandbars were counted. I detected 28 bird species, being Cygnus melancoryphus, Fulica armillata y Larus dominicanus the most numerous. The importance of carry out systematic samplings for a complete understanding of waterfowl assemblages throughout the region and for conservation actions is discussed.

Key Words: Birds, Chile, Cygnus melancoryphus, Endangered Species

\section{INTRODUCCIÓN}

Los humedales de la zona central de Chile son ecosistemas valiosos desde el punto de vista ambiental y humano dada su diversidad biológica y función ecológica (Estades et al. 2012, Estades y Vukasovic 2013). Estos ecosistemas proveen alimentación y sitios de reproducción y refugio para muchas aves silvestres debido a su alta productividad y heterogeneidad de hábitats (González et al. 2011, Cienfuegos et al. 2012). Uno de los factores que determina la riqueza y abundancia de las aves acuáticas en estos sistemas son los efectos antrópicos (Peña-Villalobos et al. 2012, Mella-Romero et al. 2018). En el sector costero de la zona central existe una gran cantidad de humedales sometidos a una fuerte influencia antrópica, donde es posible encontrar ensambles diversos de aves acuáticas (Stuardo y Valdovinos 1989, Cánepa et al. 2006, SilvaGarcía y Brewer 2007, Estades y Vukasovic 2007). En específico, para la gran mayoría de los sistemas acuáticos de la Región de O’Higgins, no se cuenta con registros de su avifauna en la literatura, o dichos registros no son periódicamente actualizados.

En este trabajo, el objetivo fue describir el ensamble de aves acuáticas presente en la laguna Cáhuil, humedal colindante al poblado de Cáhuil (Pichilemu, Cardenal Caro, Región de O’Higgins), mediante 
muestreos mensuales durante el año 2015. Además, se busca (i) comparar los resultados obtenidos con la única descripción previa de la avifauna acuática del humedal (Cánepa et al. 2006); (ii) comentar la importancia potencial de esta laguna para las dos especies de cisnes nativos, en particular para Cygnus melancoryphus (cisne de cuello negro), especie amenazada y (iii) enfatizar la necesidad de realizar muestreos periódicos en los humedales de esta zona de Chile central, para lograr un entendimiento acabado de los ensambles de avifauna acuática y para su aplicación en programas de conservación.

La laguna Cáhuil (Figura 1) es un humedal ubicado en la desembocadura del estero La Palmilla (34²7’S, 7200'O; Andrade y Grau 2005), Región del Libertador Bernardo O’Higgins, Chile central. El clima es mediterráneo con lluvias invernales, estación seca prolongada y gran nubosidad (Luebert y Pliscoff 2017). Este humedal está sometido a distintas perturbaciones antrópicas, siendo el tránsito de embarcaciones turísticas durante el verano una de las más relevantes (Andrade y Grau 2005) pues conlleva alteración de nidos, atropello de pollos, contaminación con residuos de combustible y desechos plásticos (Peña-Villalobos et al. 2012, Mella-Romero et al. 2018). Otra alteración relevante fue la creación del puente Cáhuil en 2001, que cruza toda la laguna para conectar Pichilemu y Paredones. En conjunto, estas perturbaciones caracterizan a este ecosistema, en el que se ha observado la presencia de aves acuáticas (Cánepa et al. 2006).

\section{MATERIAL Y MÉTODO}

Para determinar riqueza de especies y el número de ejemplares de aves en laguna Cáhuil, establecí un programa de monitoreo mensual durante todo el año 2015. Se establecieron cuatro transectos de muestreo (prospección terrestre) que abarcaron $200 \mathrm{~m}$ cada uno (Figura 1). Se registraron las especies presentes y su número de individuos. Las aves fueron censadas en el espejo de agua, en las barras de arena y en la ribera inmediata del humedal. Siempre que fue posible, se contabilizaron las aves de un sector desde dos puntos de muestreo dentro del transecto, con el fin de corregir por el efecto del desplazamiento de aves (precaución para evitar el recuento de ejemplares; Peña-Villalobos et al. 2012). Las observaciones se

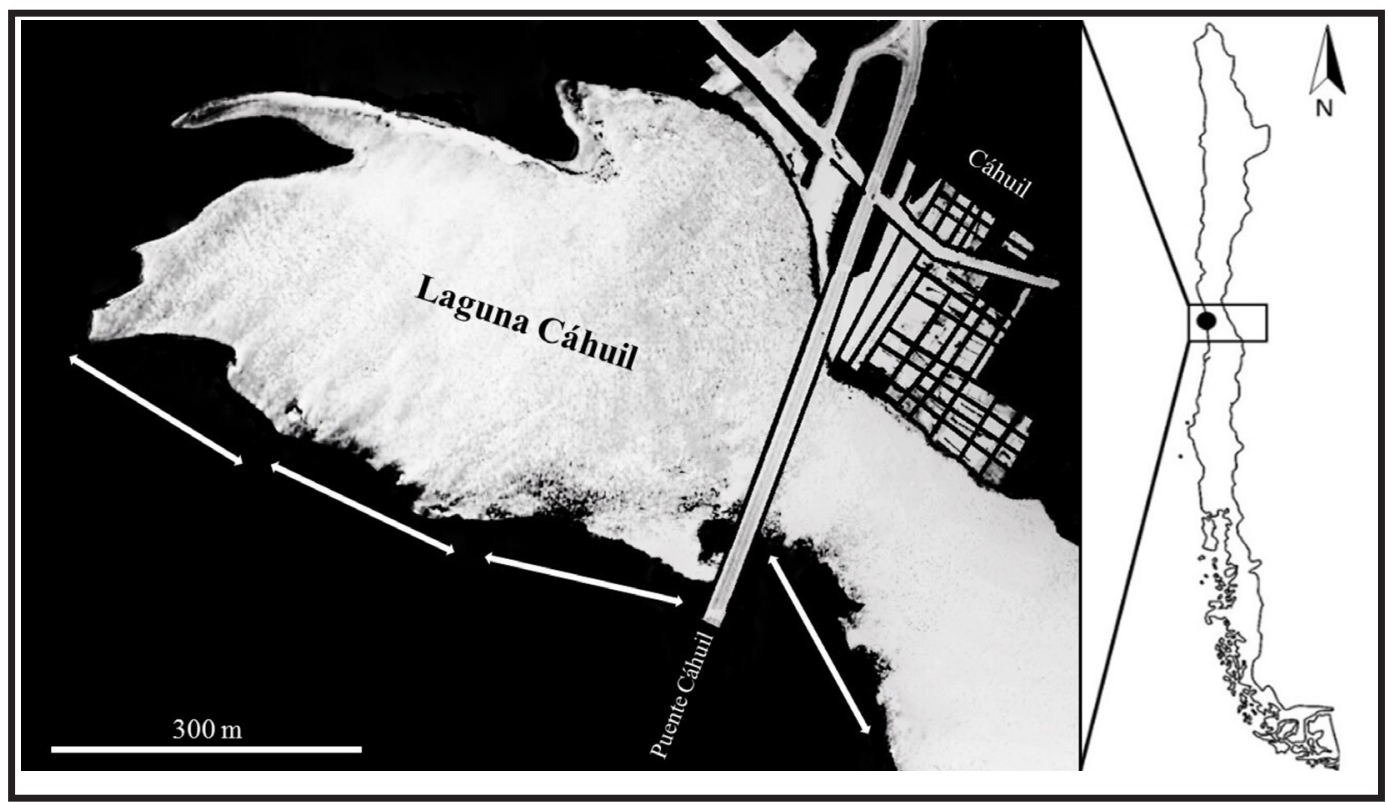

Figura 1. Laguna Cáhuil. Las flechas bidireccionales blancas $(200 \mathrm{~m})$ representan los transectos. 
realizaron de 08:00 a 11:00 horas y al mes siguiente, de 16:00 a 19:00 horas (en forma alternada cada mes, Mella-Romero et al. 2018). Los censos se llevaron a cabo empleando binoculares Celestron 10-30x50. Para la identificación de las especies de aves se utilizó la guía de campo de Jaramillo (2003) (González-Acuña et al. 2004, González et al. 2011, Peña-Villalobos et al. 2012). Los censos se llevaron a cabo usando la clasificación de Victoriano et al. (2006) en la que se consideran aves acuáticas íntegramente los órdenes Podicipediformes, Pelecaniformes, Anseriformes, Gruiformes, Charadriiformes y Suliformes. La elección anterior se justifica en que se facilitan las comparaciones entre los cuerpos de agua dentro de una misma región bioclimática (Peña-Villalobos et al. 2012, Mella-Romero et al. 2018).

\section{RESULTADOS Y DISCUSIÓN}

Durante este estudio, se registraron 28 especies de aves asociadas a la laguna Cáhuil. Las familias más representadas fueron Anatidae (seis especies), Laridae (cinco especies) y Scolopacidae (cuatro especies). Las especies más numerosas fueron el cisne de cuello negro (Cygnus melancoryphus), la tagua (Fulica armillata) y la gaviota dominicana (Larus dominicanus), las que superaron la decena de individuos durante todos los meses del año (Tabla 1). El cisne de cuello negro superó los 40 individuos durante los meses de verano (enero, febrero y marzo). Entre diciembre y marzo registré las mayores cantidades de especies y las mayores cantidades de individuos. En enero registré el mayor número de especies (22) y en marzo el mayor número de individuos (309). El mes en que se registró una menor riqueza fue julio (solo siete especies), y en el que se registró un menor número de individuos fue junio (103 ejemplares; Tabla 1).

En un estudio previo, Cánepa et al. (2006) registraron 33 especies de aves acuáticas para laguna Cáhuil, considerando como tal los mismos órdenes que en el presente trabajo y sin reportar el número de individuos de cada especie. Este es el único antecedente sobre el ensamble de la avifauna acuática en el humedal, con muestreos realizados en los meses de octubre y noviembre del año 2004. Especies coincidentes reportadas en dicho trabajo y el presente estudio fueron 22 (Tabla 1). Tanto en el artículo de Cánepa et al. (2006) como en el presente reporte, la familia más representada fue Anatidae (siete y seis especies, respectivamente). Especies registradas sólo en el trabajo de Cánepa et al. (2006) fueron: blanquillo (Podiceps occipitalis), picurio (Podilymbus podiceps), garza boyera (Bubulcus ibis), huairavo (Nycticorax nycticorax), pato rana de pico delgado (Oxyura vittata), pato gargantillo (Anas bahamensis), pidén (Pardirallus sanguinolentus), tagüita (Gallinula melanops), un gaviotín no determinado (Sterna sp.), rayador (Rynchops niger) y un playero no determinado (Calidris sp.).

Entre las aves observadas en laguna Cáhuil, detecté tres especies con prioridad de conservación (utilizando como criterio el Reglamento de Clasificación de Especies del Ministerio del Medio Ambiente): el chorlo nevado (Vulnerable), que fue avistado solo en abril y mayo (muy pocos individuos, Tabla 1), el cisne coscoroba y el cisne de cuello negro, especies consideradas como "en peligro" y "vulnerable", respectivamente (MMA 2018). El cisne de cuello negro fue observado todos los meses de muestreo, a pesar de la presencia humana constante en los bordes de la laguna (i.e., dueños de pequeños botes y turistas). El cisne de cuello negro fue más numeroso en los meses estivales (Tabla 1), coincidiendo con lo registrado por Mella-Romero et al. (2018) en laguna Petrel, cercana a laguna Cáhuil y de similares características. En esta comunicación se sugiere realizar muestreos para ambas especies de cisnes en el estero La Palmilla, dado que estos serían más abundantes aguas arriba (en donde este cuerpo de agua se ensancha y tienen lugar las Salinas de Cáhuil). Lo anterior coincidiría con lo reportado para el cisne de cuello negro por Silva-García (2018), en donde se expone que en el "estero Cáhuil" los números poblacionales de dicha especie serían elevados. En el trabajo citado se menciona "estero Cáhuil” sensu lato, muy probablemente refiriéndose al estero La Palmilla y su continuación en el estero Nilahue. 
Laguna Cáhuil forma parte de una red interconectada de humedales de la zona central y es uno de los pocos sistemas que cuenta con alguna información cuantitativa sobre ensamble de aves acuáticas en la Región de O’Higgins (Cánepa et al. 2006). En el tramo costero entre laguna Petrel (ubicada también en las cercanías de Pichilemu; Mella-Romero et al. 2018) y laguna Cáhuil (10,3 km), existen al menos cinco humedales que no cuentan con ningún registro sobre su avifauna acuática en la literatura (e.g., laguna El Barro, laguna de los Curas). Así, este trabajo buscar resaltar el sector completo como potencial sitio de relevancia ecológica para especies amenazadas (e.g., C. coscoroba, C. melancoryphus). Para lo anterior, sería necesario desarrollar muestreos periódicos en los humedales costeros de la zona, obteniendo datos de riqueza y abundancia que serían útiles para un mayor entendimiento sobre las dinámicas poblacionales y estacionales de la avifauna acuática presente. En este sentido, cabe mencionar la existencia de un Fondo Mundial para el Medio Ambiente (Global Environment Facility, GEF, en inglés) sobre Humedales Costeros que se está ejecutando en el humedal de Cáhuil actualmente (el que incluye la laguna Cáhuil, el estero La Palmilla y las Salinas de Cáhuil). A través de este proyecto, el Ministerio del Medio Ambiente busca promover la implementación de buenas prácticas y consideraciones ambientales en distintos sectores productivos de la zona, lo que se busca llevar a cabo diseñando un plan de manejo junto a la comunidad. Estas acciones pueden asociarse a la puesta en práctica de la nueva Ley de Humedales Urbanos, como herramientas sinérgicas de conservación y manejo. El presente artículo describe el ensamble de la avifauna acuática de la laguna Cáhuil durante el año 2015, y los datos aquí presentados deben ser considerados como antecedentes en la implementación del plan de manejo mencionado, en el marco del proyecto GEF que está comenzando a implementarse en Cáhuil.

\section{AGRADECIMIENTOS}

El autor agradece a Jorge Mella Ávila por su contribución en la logística de las campañas de terreno. A la Comisión Nacional de Investigación Científica y Tecnológica, Beca de Doctorado Nacional/2019-21190472. Finalmente, se agradece a Juan Carlos Torres por sus valiosos comentarios.

\section{REFERENCIAS BIBLIOGRÁFICAS}

ANDRADE, B. y S. GRAU. 2005 La laguna Cáhuil, un ejemplo de estuario estacional en Chile Central. Revista de Geografía Norte Grande 33: 59-72.

CÁNEPA, A., P. TELLO y P. MARTÍNEZ. 2006 Nota sobre la avifauna de las lagunas costeras de la VI Región. Noticiario Mensual Museo Nacional de Historia Natural 375: 3-10.

CIENFUEGOS, R., J. CAMPINO, J. GIRONÁS, R. ALMAR y M. VILLAGRÁN. 2012 Desembocaduras y Lagunas Costeras en la Zona Central de Chile. Pp. 21-65, en Fariña, J. \& A. Camaño (eds) Humedales Costeros de Chile, Aportes Científicos a su Gestión Sustentable. Ediciones UC, Chile.

ESTADES, C.F. y M.A. VUKASOVIC. 2007 Estado de conservación de las Aves de la región de O’Higgins. Libro rojo de la región de O'Higgins. Rancagua: Corporación Nacional Forestal-Universidad de Chile, 53-8.

ESTADES, C.F., M.A. VUKASOVIC y J. AGUIRRE. 2012 Aves en los Humedales Costeros de Chile. Pp. 67-99, en: Fariña, J. y A. Camaño (eds.) Humedales Costeros de Chile, Aportes Científicos a su Gestión Sustentable. Ediciones UC, Chile.

ESTADES, C.F. Y M.A. VUKASOVIC. 2013 Waterbird population dynamics at estuarine wetlands of central Chile. Ornitología Neotropical 24: 67-83.

GOBIERNO DE CHILE.2018. Nómina de especies según estado de conservación: 14º Proceso RCE. Disponible en: http://www.mma.gob.cl/clasificacionespecies/listado-especies-nativas-segun-estado-2014.htm. Consultado el 3 de febrero de 2020.

GONZÁLEZ-ACUÑA, D., C. BENAVENTE-CARMONA y R.A. FIGUEROA. 2004 Avifauna de la Laguna Santa Elena, Región del Bío-Bío. Boletín Chileno de Ornitología 10: 13-18.

GONZÁLEZ, A.L., M.A. VUKASOVIC y C.F. ESTADES. 2011 Variación temporal en la abundancia y diversidad de aves en el humedal del Río Itata, región del Bío-Bío, Chile. Gayana 75: 170-181. 
JARAMILLO, A. 2003 Birds of Chile. Princeton University Press., Princeton, 288 pp.

LUEBERT, F. y P. PLISCOFF. 2017 Sinopsis bioclimática y vegetacional de Chile: Segunda Edición. Editorial Universitaria, Santiago, Chile. 381 pp.

MELLA-ROMERO, J., I. PEÑA-VILLALOBOS y M. SALLABERRY. 2018 Aves acuáticas en Laguna Petrel (Región del Libertador Bernardo O’Higgins): abundancia y propuesta de conservación. Boletín del Museo Nacional de Historia Natural 67: 77-89.

PEÑA-VILlalobos, I., P. FIBLA, J. SAlAZAR y M. SALLABERRY. 2012 Cambios temporales en la abundancia y composición del ensamble de aves acuáticas en tranques artificiales en Chile central. Gayana 76: 92-101.

SILVA-GARCÍA, C.M. y G.L. BREWER. 2007 Breeding behavior of the Coscoroba Swan (Coscoroba coscoroba) . En: El Yali wetland, central Chile. Ornitología Neotropical 18: 573-585.

SILVA-GARCÍA, C.M. 2018 Cisne de cuello negro, Cygnus melancoryphus. Pp. 48-49, en Medrano, F., R. Barros, H.V. Norambuena, R. Matus \& F. Schmitt (eds) Atlas de las aves nidificantes de Chile. Ediciones Red de Observadores de Aves y Vida Silvestre de Chile, Chile.

STUARDO, J. y C. VALDOVINOS. 1989 Estuarios y lagunas costeras: ecosistemas importantes del Chile central. Ambiente y Desarrollo 5: 107-115.

VICTORIANO, P., A. GONZÁLEZ y R. SCHLATTER. 2006 Estado de conocimiento de las aves de aguas continentales de Chile. Gayana 70: 140-162. 
Tabla 1. Aves registradas durante 2015 en laguna Cáhuil, Región de O’Higgins, Chile central. En asterisco, especies que coinciden con las detectadas por Canepa et al. (2006).

\begin{tabular}{|c|c|c|c|c|c|c|c|c|c|c|c|c|c|}
\hline Especie & Nombre Común & 苂 & $\begin{array}{l}0 \\
0 \\
1\end{array}$ & $\sum_{\Sigma}^{\bar{\pi}}$ & 远 & $\stackrel{\vec{\Sigma}}{\mathrm{\Sigma}}$ & $\Xi$ & $\Xi$ & $\stackrel{80}{8}$ & i & $\dot{0}$ & zo & $\stackrel{0}{a}$ \\
\hline Rollandia rolland & Pimpollo* & 2 & 0 & 4 & 2 & 48 & 2 & 0 & 0 & 4 & 8 & 4 & 0 \\
\hline Podiceps major & Huala* & 2 & 4 & 0 & 0 & 0 & 0 & 0 & 5 & 0 & 0 & 0 & 0 \\
\hline Phalacrocorax brasilianus & Yeco* & 48 & 14 & 22 & 6 & 2 & 2 & 2 & 7 & 2 & 2 & 4 & 9 \\
\hline Ardea alba & Garza grande* & 2 & 2 & 0 & 2 & 0 & 0 & 0 & 1 & 1 & 1 & 2 & 2 \\
\hline Egretta thula & Garza chica* & 4 & 8 & 2 & 4 & 4 & 2 & 1 & 2 & 3 & 6 & 4 & 4 \\
\hline Anas georgica & Pato jergón grande* & 22 & 16 & 10 & 0 & 0 & 0 & 0 & 0 & 0 & 0 & 9 & 14 \\
\hline Anas sibilatrix & Pato real* & 0 & 2 & 0 & 0 & 0 & 0 & 0 & 0 & 0 & 0 & 0 & 0 \\
\hline Anas cyanoptera & Pato colorado* & 2 & 0 & 4 & 0 & 0 & 0 & 0 & 0 & 0 & 0 & 0 & 0 \\
\hline Spatula platalea & Pato cuchara* & 4 & 0 & 2 & 0 & 0 & 0 & 0 & 0 & 0 & 0 & 0 & 4 \\
\hline Coscoroba coscoroba & Cisne coscoroba* & 4 & 6 & 2 & 2 & 0 & 0 & 0 & 58 & 0 & 0 & 4 & 4 \\
\hline Cygnus melancoryphus & Cisne de cuello negro* & 42 & 84 & 66 & 25 & 20 & 28 & 29 & 30 & 20 & 10 & 18 & 32 \\
\hline Fulica armillata & Tagua* & 22 & 66 & 44 & 33 & 21 & 31 & 30 & 27 & 15 & 18 & 18 & 20 \\
\hline Fulica leucoptera & Tagua chica* & 0 & 0 & 23 & 5 & 2 & 0 & 0 & 0 & 0 & 0 & 0 & 0 \\
\hline Fulica rufifrons & Tagua de frente roja & 2 & 2 & 0 & 0 & 0 & 0 & 0 & 0 & 0 & 0 & 0 & 0 \\
\hline Himantopus mexicanus & Perrito* & 4 & 6 & 8 & 2 & 2 & 0 & 0 & 5 & 0 & 0 & 0 & 2 \\
\hline Vanellus chilensis & Queltehue* & 3 & 1 & 0 & 0 & 0 & 0 & 0 & 2 & 0 & 1 & 0 & 0 \\
\hline Charadrius collaris & Chorlo de collar & 0 & 0 & 0 & 2 & 2 & 4 & 0 & 0 & 0 & 0 & 0 & 0 \\
\hline Charadrius nivosus & Chorlo nevado & 0 & 0 & 0 & 2 & 3 & 0 & 0 & 0 & 0 & 0 & 0 & 0 \\
\hline Haematopus palliatus & Pilpilén común* & 15 & 10 & 8 & 7 & 10 & 0 & 10 & 22 & 18 & 32 & 8 & 24 \\
\hline Larus dominicanus & Gaviota dominicana* & 44 & 12 & 42 & 22 & 14 & 16 & 28 & 45 & 40 & 45 & 12 & 10 \\
\hline Chroicocephalus maculipennis & Gaviota cáhuil* & 4 & 2 & 44 & 22 & 13 & 18 & 10 & 21 & 14 & 12 & 6 & 3 \\
\hline Leucophaeus modestus & Gaviota garuma & 0 & 0 & 0 & 0 & 0 & 0 & 0 & 22 & 0 & 0 & 0 & 0 \\
\hline Leucophaeus pipixcan & Gaviota Franklin & 14 & 0 & 4 & 0 & 0 & 0 & 0 & 0 & 4 & 10 & 22 & 98 \\
\hline Sterna trudeaui & Gaviotín piquerito & 0 & 0 & 4 & 2 & 0 & 0 & 0 & 0 & 0 & 2 & 0 & 0 \\
\hline Tring a flavipes & Pitotoy chico* & 2 & 4 & 12 & 0 & 0 & 0 & 0 & 0 & 0 & 2 & 0 & 4 \\
\hline Tringa melanoleuca & Pitotoy grande* & 2 & 2 & 5 & 0 & 0 & 0 & 0 & 0 & 0 & 1 & 0 & 6 \\
\hline Numenius phaeopus & Zarapito* & 8 & 0 & 0 & 0 & 0 & 0 & 0 & 0 & 0 & 1 & 2 & 12 \\
\hline Calidris bairdii & Playero de Baird* & 5 & 7 & 3 & 0 & 0 & 0 & 0 & 0 & 0 & 0 & 0 & 2 \\
\hline \multicolumn{2}{|c|}{ Número de especies } & 22 & 18 & 19 & 15 & 12 & 8 & 7 & 13 & 10 & 15 & 13 & 17 \\
\hline \multicolumn{2}{|c|}{ Número de individuos } & 257 & 248 & 309 & 138 & 141 & 103 & 110 & 247 & 121 & 151 & 113 & 250 \\
\hline
\end{tabular}

